\title{
Circuit Optimization Study According to the Maximum Principle
}

\author{
ALEXANDER ZEMLIAK \\ Department of Physics and Mathematics \\ Autonomous University of Puebla \\ Av. San Claudio y 18 Sur, Puebla, 72570 \\ MEXICO \\ Institute of Technical Physics \\ National Technical University of Ukraine \\ UKRAINE
}

\begin{abstract}
The minimization of the processor time of designing can be formulated as a problem of time minimization for transitional process of dynamic system. A special control vector that changes the internal structure of the equations of optimization procedure serves as a principal tool for searching the best strategies with the minimal CPU time. In this case a well-known maximum principle of Pontryagin is the best theoretical approach for finding of the optimum structure of control vector. Practical approach for realization of the maximum principle is based on the analysis of behavior of a Hamiltonian for various strategies of optimization. The possibility of applying the maximum principle to the problem of optimization of electronic circuits is analyzed. It is shown that in spite of the fact that the problem of optimization is formulated as a nonlinear task, and the maximum principle in this case isn't a sufficient condition for obtaining a minimum of the functional, it is possible to obtain the decision in the form of local minima. The relative acceleration of the CPU time for the best strategy found by means of maximum principle compared with the traditional approach is equal two to three orders of magnitude.
\end{abstract}

Key-Words: - Circuit optimization, control theory, controllable dynamic system, optimization strategies, maximum principle.

Received: June 14, 2021. Revised: October 29, 2021. Accepted: November 13, 2021. Published: December 9, 2021.

\section{Introduction}

To improve the overall quality of electronic circuit designs, it is very important to reduce their design time. Some works devoted to this problem focus on how to reduce the number of operations when solving two main problems: circuit analysis and numerical optimization. By solving these problems successfully, one can reduce the total time required for analog circuit optimization and this fact serves as a basis for improving design quality.

The methods used to analyze complex systems are being improved continuously. Some methods reduce the time need for circuit analysis. This includes the well-known idea of using sparse matrix methods [1-2] and decomposition methods [3]. Some alternative methods such as homotopy methods [4] were successfully applied to circuit analysis.

The techniques for analog circuit optimization can be classified in two main groups: deterministic optimization algorithms and stochastic search algorithms. Practical methods of optimization were developed for circuit designing, timing, and area optimization [5-6]. However, classical deterministic optimization algorithms may have a number of drawbacks: they may require that a good initial point be selected in the parameter space, they may reach an unsatisfactory local minimum, and they require that the cost function be continuous and differentiable. To overcome these issues, special methods were applied to determine the initial point of the process by centering [7] or applying geometric programming methods [8].

Stochastic search algorithms, especially evolutionary computation algorithms like genetic algorithms, differential evaluation, genetic programming, particle swarm optimization, etc. have been developed in recent years [9-15]. Genetic algorithms have been employed as optimization routines for analog circuits due to the ability to find a satisfactory solution. A special algorithm defined as a particle swarm optimization technique is one of the evolutionary algorithms and competes with genetic algorithms. This method is successfully used for electromagnetic problems and for optimization of microwave systems [16-17]. 
A more general formulation of the circuit optimization problem was developed on a heuristic level some decades ago [18]. This approach ignored Kirchhoff's laws for all or part of a circuit during the optimization process. The practical aspects of this idea were developed for the optimization of microwave circuits [19] and for the synthesis of high-performance analog circuits [20] in an extreme case where all the equations of the circuit were not solved during the optimization process.

In work [21] the problem of circuit optimization is formulated in terms of the theory of optimal control. Thus, the process of circuit optimization was generalized and defined as the dynamic controllable system. In this case, the basic element is the control vector that changes the structure of the equations of the system of optimization process. Thus, there is a set of strategies of optimization that have different number of operations and different computing times. At the same time, the problem of searching for the optimal strategy and the corresponding optimal trajectory can be solved most appropriately within the Pontryagin maximum principle [22].

The main complexity of application of the maximum principle consists of the search of initial values for auxiliary variables at the solution of the conjugate system of equations. Application of the maximum principle in case of linear dynamic systems is based on the iterative process [23-24].

In case of nonlinear systems, the convergence of this process is not guaranteed. However, application of the additional approximating procedures [25-26] allows constructing sequence of the solutions converging to a limit under certain conditions.

The first step in the problem of possibility of application of maximum principle for circuit optimization was presented in [27] for twodimensional case. In the present work, the solution of the problem is presented for $N$-dimensional case.

\section{Problem Formulation}

In accordance with the conventional approach, the process of electronic circuit optimization is defined as the problem of minimizing an objective function $C(\mathbf{X}), \mathbf{X} \in R^{N}$, with constraints given by a system of the circuit's equations based on Kirchhoff's laws. We assume that, by minimizing $C(\mathbf{X})$, we achieve all our design goals. The circuit optimization problem can be generalized by introducing a special control vector $\mathbf{U}=\left(u_{1}, u_{2}, \ldots, u_{M}\right)$ and a special generalized objective function $F(\mathbf{X}, \mathbf{U})$. The electronic circuit optimization process can be defined as the problem of minimizing the generalized objective function $F(\mathbf{X}, \mathbf{U})$ based on the vector equation (1) with the constraints (2). The system of constraints is the mathematical model of the electronic circuit.

$$
\begin{gathered}
\mathbf{X}^{s+1}=\mathbf{X}^{s}+t_{s} \mathbf{H}^{s}, \\
\left(1-u_{j}\right) g_{j}(\mathbf{X})=0, j=1,2, \ldots, M,
\end{gathered}
$$

where $\mathbf{X} \in R^{N}, \mathbf{X}=\left(\mathbf{X}^{\prime}, \mathbf{X}^{\prime \prime}\right), \mathbf{X}^{\prime} \in R^{K}$ is a vector of independent variables, $\mathbf{X}^{\prime \prime} \in R^{M}$ is a vector of dependent variables, $M$ is the number of the circuit's dependent variables, $K$ is the number of independent variables, $N$ is the total number of variables $(N=K+M)$ and $t_{s}$ is an iteration parameter. The equation (1) describes a two-step minimization procedure, and the function $H \equiv H(\mathbf{X}, \mathbf{U})$ determines the direction in which the generalized objective function $F(\mathbf{X}, \mathbf{U})$ decreases. The functions $g_{j}(\mathbf{X})$ for all $j$ define the equations of the circuit model. The components of control vector $\mathbf{U}$ are a set of control functions: $\mathbf{U}=\left(u_{1}, u_{2}, \ldots, u_{M}\right)$, where $u_{j} \in \Omega, \quad \Omega=\{0 ; 1\}$. The vector $\mathbf{U}$ leads to redistribution of expenses of computing time between the block of procedure of optimization (1) and the block of the analysis of the scheme (2). The complete set of different optimization strategies (structural basis) includes $2^{M}$ strategies. The generalized objective function $F(\mathbf{X}, \mathbf{U})$ can be defined, for example, as follows:

$$
F(\mathbf{X}, \mathbf{U})=C(\mathbf{X})+\varphi(\mathbf{X}, \mathbf{U}),
$$

where $C(\mathbf{X})$ is a non-negative ordinary objective function of the optimization process and $\varphi(\mathbf{X}, \mathbf{U})$ is a penalty function. The structure of the penalty function must potentially include all the equations from the system (2) and can be defined, for example, as follows:

$$
\varphi(\mathbf{X}, \mathbf{U})=\frac{1}{\delta} \sum_{j=1}^{M} u_{j} g_{j}^{2}(\mathbf{X})
$$

where $\delta$ is an additional coefficient used to adapt the penalty function. In our context, $\delta$ equals 1 .

This definition of the circuit optimization problem allows us to redistribute the computing time between the problems (1) and (2). A control function $u_{j}$ has the following meaning: if $u_{j}=0$, 
the $j$ th equation is present in the system (2) and the term $g_{j}^{2}(\mathbf{X})$ is removed from the equation (4); and, the other way around, if $u_{j}=1$, the $j$ th equation is removed from the system (2) and the term $g_{j}^{2}(\mathbf{X})$ is present in the equation (4). We can define two special strategies: $\mathbf{U}=(0,0, \ldots, 0)$ and $\mathbf{U}=(1,1, \ldots, 1)$. The first strategy can be named as Traditional Strategy of Optimization (TSO) and corresponds to the solution of system (2) at each point of optimization process. The second strategy can be named as Modified Traditional Strategy of Optimization (MTSO) and corresponds to the elimination of the system (2), but in this case all the information on the circuit is included in the penalty function (4). The vector $\mathbf{U}$ is the main tool of this methodology: it controls the dynamic process of minimizing the objective functions $F(\mathbf{X}, \mathbf{U})$ and $C(\mathbf{X})$ in the possible minimum time. This definition allows us to express the problem of searching for the optimal strategy as the typical problem of minimizing a functional, where the functional is the CPU time. When defining the optimization process as a dynamical system, a more standard approach is to use differential equations, in continuous form. We can rewrite the main system of the optimization procedure (1) in continuous form as the following system of differential equations:

$$
\frac{d x_{i}}{d t}=f_{i}(\mathbf{X}, \mathbf{U}), \quad i=1,2, \ldots, N,
$$

Together with the equations (2), (3) and (4), this system specifies the continuous form of the optimization process. The structure of the functions $f_{i}(\mathbf{X}, \mathbf{U})$ is defined by a concrete optimization method. For example, for the gradient method, it takes the following form:

$$
\begin{gathered}
f_{i}(\mathbf{X}, \mathbf{U})=-\frac{\delta}{\delta x_{i}} F(\mathbf{X}, \mathbf{U}), i=1,2, \ldots, K \\
f_{i}(\mathbf{X}, \mathbf{U})=-u_{i-K} \frac{\delta}{\delta x_{i}} F(\mathbf{X}, \mathbf{U})+\left(1-u_{i-K}\right) \alpha_{i}, i=K+1, \ldots, N,
\end{gathered}
$$

where $\alpha_{i}$ is the additional parameter defining an increment of the value of the dependent variables $x_{i}$ in the course of optimization and computed by the formula $\alpha_{i}=\frac{1}{\tau}\left[\eta_{i}\left(\mathbf{x}^{s+1}\right)-x_{i}^{s}\right], \quad$ and $\quad \eta_{i}(\mathbf{x})$ is the implicit function defining the component number $i$ of a vector $\mathbf{X}$ at the solution of system (2), $\tau$ is the step of integration and $s$ is the step number of the procedure of numerical integration of system (5). The operator $\delta / \delta x_{i}$ is defined by the formula $\frac{\delta}{\delta x_{i}} \rho(\mathbf{X})=\frac{\partial \rho(\mathbf{X})}{\partial x_{i}}+\sum_{p=K+1}^{K+M} \frac{\partial \rho(\mathbf{X})}{\partial x_{p}} \frac{\partial x_{p}}{\partial x_{i}} \quad$ and determines the application of the gradient method for a complex function that has both independent and dependent variables.

In the present work, the gradient method was used as the main method of optimization. However, the application of a gradient method is not a restriction of the proposed approach. It is possible to embed any concrete method of optimization in the proposed methodology. All main properties of the proposed approach remain fair for any concrete optimization method.

The similar formulation of a problem of optimization can be used not only for electronic systems, but also for any physical object if the mathematical model of an object can be presented by system of the nonlinear algebraic equations.

By using formulas (2)-(6), we formulate the circuit optimization process as a controllable process or as a controllable dynamical system. The vector $\mathbf{U}$ defines the right hand parts of the system (5) and gives the possibility of changing the optimization strategies and operation's number. Such approach contains optimization strategies much more optimum than TSO. So, the vector $\mathbf{U}$ is the principal tool for searching and constructing the optimization process with a minimal computing time. Control functions $u_{j}$, so and functions $f_{i}(\mathbf{X}, \mathbf{U})$ are piecewise continuous. The optimal control problem for the system (5) with the noncontinuous right hand parts can be solved most correctly using Pontryagin maximum principle. The direct application of this principle is rather difficult because of nonlinear formulation of the problem of circuit optimization. The first step in the problem of possibility of application of maximum principle for circuit optimization was presented in [29] for the simplest circuit. The analytical solution was obtained in this case. The interesting question is whether it is possible to extend the obtained analytical result to the numerical solution of the optimization problem for nonlinear circuit of any dimension. The next section is devoted to this problem.

\section{Maximum Principle Application}

We need to extend earlier obtained result on the solution of $\mathrm{N}$-dimensional problem of circuit optimization. Let's consider the problem of 
optimization of a nonlinear circuit with two nodes shown on Fig1.

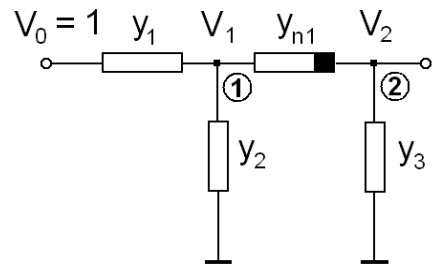

Fig. 1 Nonlinear two-node voltage divider

The given circuit is a nonlinear divider of voltage. There are three independent parameters $(K=3)$ and two dependent ones $(M=2)$. The nonlinear element has the following dependency: $y_{n 1}=y_{0}+a\left(V_{1}-V_{2}\right)^{2}$. Here and further, all physical variables are presented in the normalized, unitless form. We define the voltage $V_{0}$ as 1 , and the variables $x_{1}, x_{2}, x_{3}, x_{4}$ and $x_{5}$ as: $x_{1}^{2}=y_{1}$, $x_{2}^{2}=y_{2}, x_{3}^{2}=y_{3}, \quad x_{4}=V_{1}$, and $x_{5}=V_{2}$. By defining the components $x_{1}, x_{2}, x_{3}$ using the above formulas, we automatically obtain positive values of the conductance, which eliminates the issue of positive definiteness for each resistance and conductance and allows us to carry out optimization in the full space of the values of these variables without any restrictions. The vector of the phase variables of the circuit is $X \in R^{5}$. In this case the nonlinear element is defined by the following expression: $y_{n 1}=y_{0}+a\left(x_{4}-x_{5}\right)^{2}$.

Let's determine function of the purpose of process of optimization by the formula:

$$
C(\mathbf{X})=\left(x_{5}-w\right)^{2},
$$

where $w-$ the required value of output voltage. The model of a circuit is defined by the following system:

$$
\begin{aligned}
& g_{1}(\mathbf{X}) \equiv\left(1-x_{4}\right) x_{1}^{2}-\left\lfloor y_{0}+d\left(x_{4}-x_{5}\right)^{2} \mid\left(x_{4}-x_{5}\right)-x_{4} x_{2}^{2}=0\right. \\
& g_{2}(\mathbf{X}) \equiv\left\lfloor y_{0}+a\left(x_{4}-x_{5}\right)^{2}\right\rfloor\left(x_{4}-x_{5}\right)-x_{5} x_{3}^{2}=0
\end{aligned}
$$

The system of equations of the optimization procedure is presented by the system (5) with right hand parts (6).

We formulate the problem of circuit optimization as a task of search of the optimization strategy with a minimum possible CPU time. For this purpose, we define the functional, which is subject to minimization, by the following expression:

$$
J=\int_{0}^{T} f_{0}(\mathbf{X}) d t
$$

where $f_{0}(\mathbf{X})$ is the function that is conditionally determining the density of a number of arithmetic operations in a unit of time $t$. In that case, the integral (9) defines total number of operations necessary for circuit optimisation and is proportional to the total CPU time.

The structure of function $f_{0}(\mathbf{X})$ cannot be defined. However, we can compute CPU time using the possibilities of the compiler. We will further identify the integral (9) with CPU time, and therefore, the problem of minimization of CPU time corresponds to a problem of minimization of the integral (9).

Let's obtain the main expressions corresponding to the maximum principle. The conjugate system of the equations for the additional variables $\psi_{i}$ has a form:

$$
\frac{d \psi_{i}}{d t}=-\sum_{k=1}^{N} \frac{\partial f_{k}(\mathbf{X}, \mathbf{U})}{\partial x_{i}} \cdot \psi_{k}
$$

The Hamiltonian is determined by the following formula:

$$
\begin{aligned}
H(\mathbf{X}, \boldsymbol{\Psi}, \mathbf{U}) & =\sum_{i=1}^{N} \psi_{i} \cdot f_{i}(\mathbf{X}, \mathbf{U})= \\
& \sum_{i=1}^{K} \psi_{i} \cdot f_{i}(\mathbf{X}, \mathbf{U})+\sum_{i=K+1}^{N} \psi_{i} \cdot f_{i}(\mathbf{X}, \mathbf{U})
\end{aligned}
$$

where the first and second sums are defined by the following expressions:

$$
\begin{aligned}
& \sum_{i=1}^{K} \psi_{i} \cdot f_{i}(\mathbf{X}, \mathbf{U})=-\sum_{i=1}^{K} \psi_{i} \frac{\delta C}{\delta x_{i}} \\
&-\sum_{i=1}^{K} \psi_{i} \sum_{k=1}^{M} u_{k} \cdot \frac{\delta\left(g_{k}(\mathbf{X})\right)^{2}}{\delta x_{i}} \\
& \sum_{i=K+1}^{N} \psi_{i} \cdot f_{i}(\mathbf{X}, \mathbf{U})=\sum_{i=K+1}^{N}\left(1-u_{i-K}\right) \psi_{i} \alpha_{i} \\
&-\sum_{i=K+1}^{N} u_{i-K} \psi_{i}\left[\frac{\delta C}{\delta x_{i}}+\sum_{k=1}^{M} u_{k} \frac{\delta\left(g_{k}(\mathbf{X})\right)^{2}}{\delta x_{i}}\right]
\end{aligned}
$$


As a result the Hamiltonian can be expressed as follows:

$$
H(\mathbf{X}, \Psi, \mathbf{U})=h_{c}+h_{0}+h_{1}+h_{2},
$$

where $h_{\mathrm{c}}$ is the part of a Hamiltonian that does not depend on the control vector,

$$
h_{c}(\mathbf{X}, \boldsymbol{\Psi})=-\sum_{i=1}^{K} \psi_{i} \cdot \frac{\delta C}{\delta x_{i}}+\sum_{i=K+1}^{N} \psi_{i} \alpha_{i}
$$

Other components of a Hamiltonian depend on the control vector $\mathbf{U}$ :

$$
\begin{gathered}
h_{0}(\mathbf{X}, \boldsymbol{\Psi}, \mathbf{U})=-\sum_{i=K+1}^{N} u_{i-K} \psi_{i} \alpha_{i}, \\
h_{1}(\mathbf{X}, \boldsymbol{\Psi}, \mathbf{U})=-\sum_{i=1}^{K} \psi_{i} \sum_{k=1}^{M} u_{k} \cdot \frac{\delta\left(g_{k}(\mathbf{X})\right)^{2}}{\delta x_{i}} \\
h_{2}(\mathbf{X}, \boldsymbol{\Psi}, \mathbf{U})=-\sum_{i=K+1}^{N} u_{i-K} \psi_{i}\left[\frac{\delta C}{\delta x_{i}}+\sum_{k=1}^{M} u_{k} \frac{\delta\left(g_{k}(\mathbf{X})\right)^{2}}{\delta x_{i}}\right]
\end{gathered}
$$

Let's designate the sum of these three components as $h_{v}(\mathbf{X}, \Psi, \mathbf{U}) \quad\left(h_{\mathrm{v}}=h_{0}+h_{1}+h_{2}\right)$. Formulas (5)-(17) define the process of system optimization and the process of computing a Hamiltonian in case of a $K$ independent variable and $M$ dependent variables. In the case of the circuit presented in Figure 1, formulas are used for $K=3$ and $M=2$. In this case the control vector of $\mathbf{U}$ contains two components $\left(u_{1}, u_{2}\right)$. The supremum of the function $H(\mathbf{X}, \Psi, \mathbf{U})$ in the parameter $\mathbf{U}$ will be designated $H_{\max }$ :

$$
H_{\max }(\mathbf{X}, \boldsymbol{\Psi})=h_{c}(\mathbf{X}, \boldsymbol{\Psi})+\sup _{u \in \mathbf{U}} h_{v}(\mathbf{X}, \boldsymbol{\Psi}, \mathbf{U})
$$

For the circuit on Figure 1 this function is defined by the following expression:

$$
\begin{aligned}
H_{\max }(\mathbf{X}, \boldsymbol{\Psi})=h_{c}(\mathbf{X}, \boldsymbol{\Psi})+ \\
+\max \left\{\begin{array}{l}
h_{v}(\mathbf{X}, \boldsymbol{\Psi},(0,0)), h_{v}(\mathbf{X}, \boldsymbol{\Psi},(0,1)), \\
h_{v}(\mathbf{X}, \boldsymbol{\Psi},(1,0)), h_{v}(\mathbf{X}, \boldsymbol{\Psi},(1,1))
\end{array}\right\}
\end{aligned}
$$

The structure of the control vector providing this maximum in each point of optimization process represents the result of the use of the maximum principle. This optimal structure ensures the minimal value of the functional (9) and the minimal computing time.

\section{Numerical Results and Discussion}

The analysis of the process of optimization for a circuit with two nodes allows for the finding of the optimum structure of the control vector. The possibility of applying the maximum principle of Pontryagin to the problem of optimization of electronic circuits is analyzed. It is shown that in spite of the fact that the problem of optimization is formulated as a nonlinear task, and the maximum principle in this case isn't a sufficient condition for obtaining a maximum of the functional, it is possible to obtain the decision in the form of local minima. Local minima of the functional, which is defined as the processor time necessary for the procedure of optimization, provide a rather low value of the functional. The relative acceleration of the CPU time for the best strategy found by means of maximum principle compared with the traditional approach is equal two to three orders of magnitude.

The behaviour of Hamiltonian for four possible options of the control vector $\mathbf{U}$ : (00), (01), (10), and (11), with the correct initial value of an auxiliary vector $\boldsymbol{\Psi},\left(\boldsymbol{\Psi}_{0 c}=(0.3,-1.85,-0.35,-1.9,0.32,2.5)\right)$ is presented in Fig2.

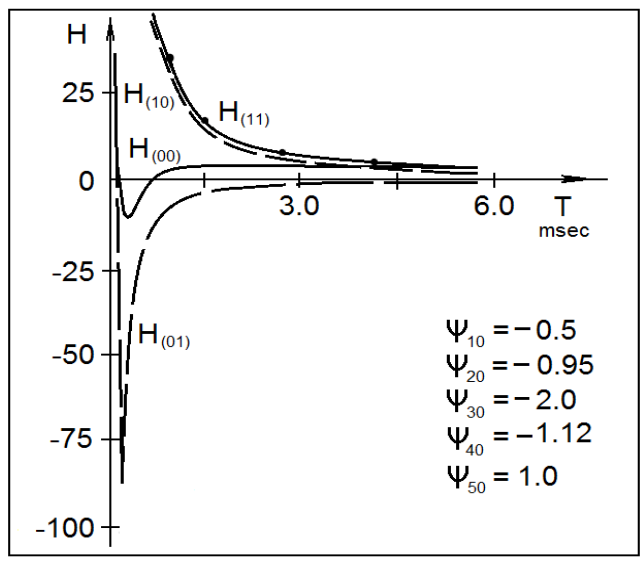

Fig. 2 Time dependency of functions $H_{(00)}, H_{(01)}$, $H_{(10)}, H_{(11)}$ for correct value of parameter $\boldsymbol{\Psi}_{0 c}$

The value of $\boldsymbol{\Psi}_{0 c}$ has been obtained by the additional optimizing procedure on the basis of a gradient method for the following initial point in process of designing $\mathbf{X}^{0}:\left(x_{10}=1.0, x_{20}=1.0, x_{30}\right.$ $\left.=1.0, \quad x_{40}=-1.5, \quad x_{50}=-1.6\right)$. Four possible combinations of the components of the control vector $\mathbf{U}$ define four various dependencies for Hamiltonian: $\quad\left(H_{(00)}, H_{(01)}, H_{(10)}, H_{(11)}\right)$. The Hamiltonian corresponding to the control vector $\mathbf{U}=(11)$ has the greatest value of all possible. 
Therefore, the optimum trajectory corresponds to this vector and defines the first part of a trajectory in the space of parameters. Some two-dimensional projections of a trajectory of optimization process in the space of variables $\mathbf{X}$ are presented in Fig3.

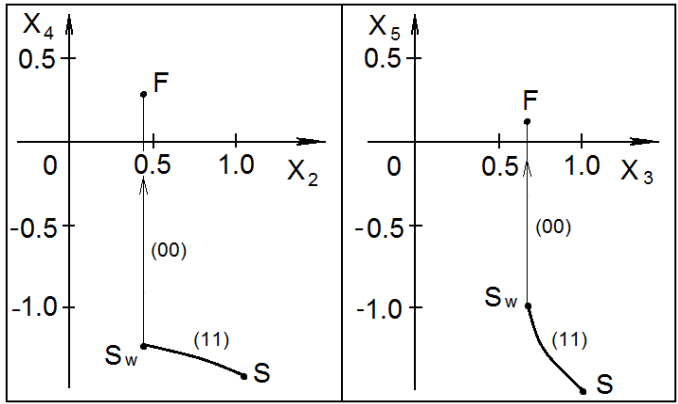

Fig. 3 Projections of trajectory of optimization process with initial point $\mathbf{X}^{0}$ :

$\left(x_{10}=1, x_{20}=1, x_{30}=1, x_{40}=-1.5, x_{50}=-1.6\right)$

A part of the trajectory from point $S$ to point $S_{\text {w }}$ corresponds to the control vector (11). Then, at a time point of $5.753 \mathrm{msec}$, which corresponds to 121 steps of integration of the system (5), the Hamiltonian corresponding to the strategy with the control vector (00) becomes the greatest of all, and at this moment the vector (00) becomes the optimum control vector. The trajectory includes the jump and the current point of the optimization process instantly moves to the final point of the solution of a problem of $F$. This effect was named as a special effect of acceleration of the circuit optimization process.

The data of optimization process for the presented circuit for four strategies of structural basis and the same initial point $\mathbf{X}^{0}$ are provided in Table 1 for comparison.

Table 1. Data of all strategies of structural basis.

\begin{tabular}{|r|r|r|r|}
\hline $\mathrm{N}$ & $\begin{array}{l}\text { Control } \\
\text { vector }\end{array}$ & $\begin{array}{l}\text { Iterations } \\
\text { number }\end{array}$ & $\begin{array}{l}\text { Total CPU } \\
\text { time }(\mathrm{sec})\end{array}$ \\
\hline 1 & $\left(\begin{array}{ll}0 & 0\end{array}\right)$ & 116973 & 16.081 \\
\hline 2 & $\left(\begin{array}{ll}0 & 1\end{array}\right)$ & 139143 & 8.897 \\
\hline 3 & $\left(\begin{array}{ll}1 & 0\end{array}\right)$ & 133154 & 11.241 \\
\hline 4 & $\left(\begin{array}{ll}1 & 1\end{array}\right)$ & 170953 & 7.934 \\
\hline
\end{tabular}

All the strategies provide the same solution for the independent variables $x_{1}, x_{2}, x_{3}$, minimizing the objective function $C(\mathbf{X})$, but they have the different iteration numbers and different total CPU time.

The most rapid strategy is MTSO, which corresponds to the control vector (11) has a processor time of $7.934 \mathrm{sec}$. Acceleration for the obtained optimum strategy in comparison with MTSO with control vector (11) of Table 1 is equal to 1,379 times and 2,795 times in comparison with TSO with control vector (00).

The behaviour of the Hamiltonian that corresponds to another choice for the initial point of optimization process of circuit $\mathbf{X}^{0}$ : $\left(x_{10}=1.0, x_{20}=\right.$ 1.0, $\left.x_{30}=1.0, x_{40}=-2.5, x_{50}=-2.5\right)$ for four possible values of the control vector $\mathbf{U}$ : (00), (01), (10), and (11) is shown in Fig4.

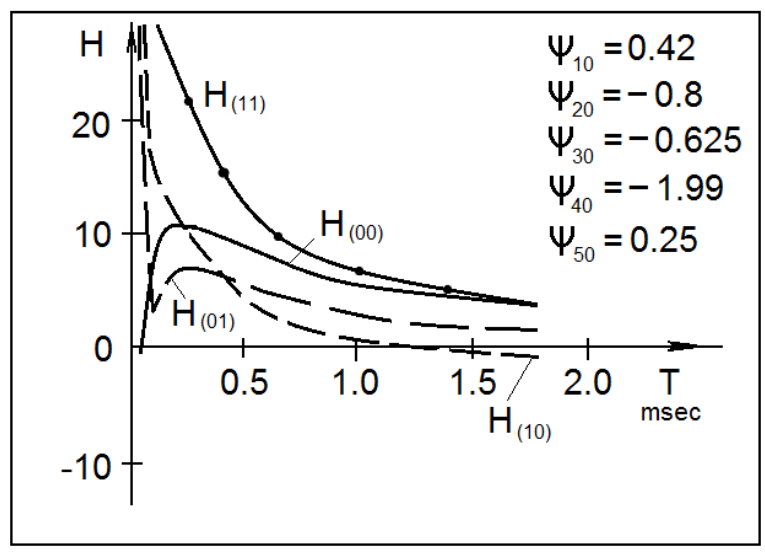

Fig. 4 Time dependency of functions $H_{(00)}, H_{(01)}$, $H_{(10)}, H_{(11)}$ for other correct value of parameter $\boldsymbol{\Psi}_{0 c}=(0.42,-0.8,-0.625,-1.99,0.25)$

In this case the following correct value of an auxiliary vector $\quad \Psi_{0 c}=(0.42,-0.8,-0.625,-1.99,0.25)$ providing the minimum value of processor time has been obtained. In this example, the optimization procedure is defined by the control vector (11) from $\mathrm{T}=0$ to $\mathrm{T}=1.761 \mathrm{msec}$ because the Hamiltonian of $H_{(11)}$ has the maximum value for this control vector of the four possible. Then, at a time of $\mathrm{T}=1.761$ msec, which corresponds to the 35th step of process of integration of system (5) the Hamiltonian corresponding to the control vector (00) has the maximum value $\left(H_{(00)}>=H_{(11)}\right)$ and the switching to the TSO is observed. The movement corresponding to the strategy (00) is carried out on one step of integration and the current point of the optimization process moves to the final point of $F$ with the given accuracy. It is clear from the behaviour of the projections of the optimization trajectory shown in Fig5.

It is important to emphasize that the numerical algorithm automatically switches from one strategy to another on the basis of ratio (19), corresponding to the maximum principle. 


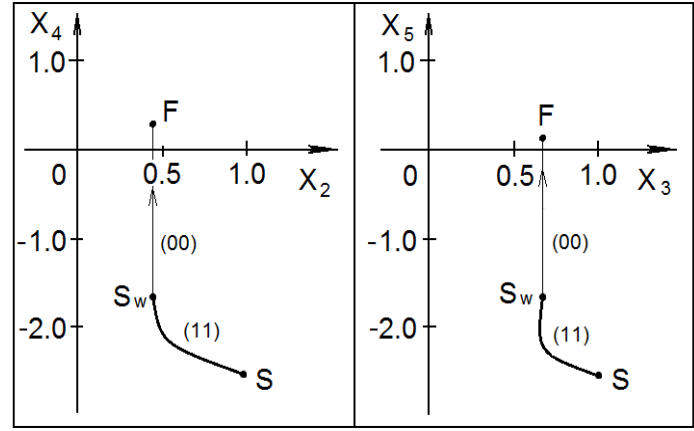

Fig. 5 Projections of trajectory of optimization process with initial point $\mathbf{X}^{0}$ :

$\left(x_{10}=1, x_{20}=1, x_{30}=1, x_{40}=-2.5, x_{50}=-2.5\right)$

It would be desirable to note that the obtained decision is not the only local minimum of the target function of (9). Other local minimum has been reached with the other vector $\boldsymbol{\Psi}$.

The behaviour of the function of Hamilton for the same initial point of the optimization process of the circuit $\mathbf{X}^{0}:\left(x_{10}=1, x_{20}=1, x_{30}=1, x_{40}=-2.5, x_{50}=-2.5\right)$ but containing other initial value for auxiliary vector $\boldsymbol{\Psi}$ is presented in Fig6. The correct value of $\boldsymbol{\Psi}_{0 c}$ obtained by the additional optimizing procedure is next $\boldsymbol{\Psi}_{0 c}=(0.1,-0.02,-0.5,-0.2,0.6)$. In this case the other strategy is optimal one.

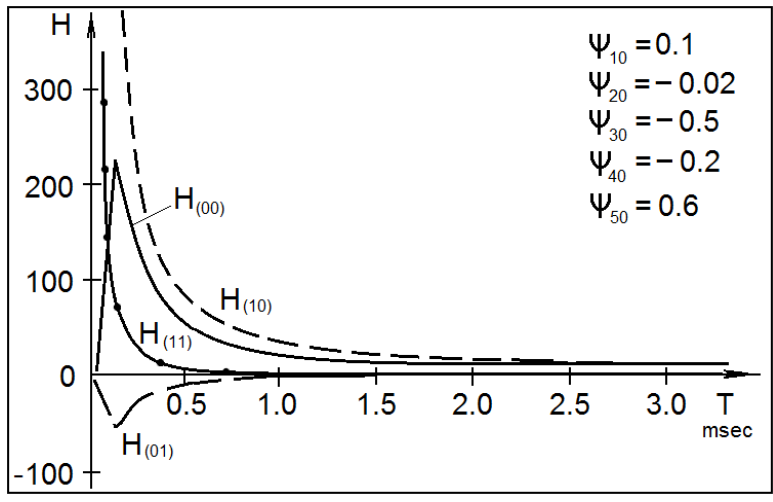

Fig. 6 Time dependency of functions $H_{(00)}, H_{(01)}$, $H_{(10)}, H_{(11)}$ for other correct value of parameter

$$
\boldsymbol{\Psi}_{0 c}=(0.1,-0.02,-0.5,-0.2,0.6)
$$

An additional optimization by means of parameter $\boldsymbol{\Psi}_{0}$ leads to other local minimum that is visible from the obtained dependencies. The Hamiltonian corresponding to the strategy with control vector (10) accepts the maximum value and this strategy is the first part of the optimum strategy. The Hamiltonian corresponding to TSO with the control vector (00) is greater than for all other strategy from the point corresponding to the 38th step of integration, and a switching to TSO takes place. That is also clear from the behaviour of projections of the optimization trajectory in Fig7.

The total time of optimization is equal 3.345 msec. This decision represents other local minimum of the functional (9).

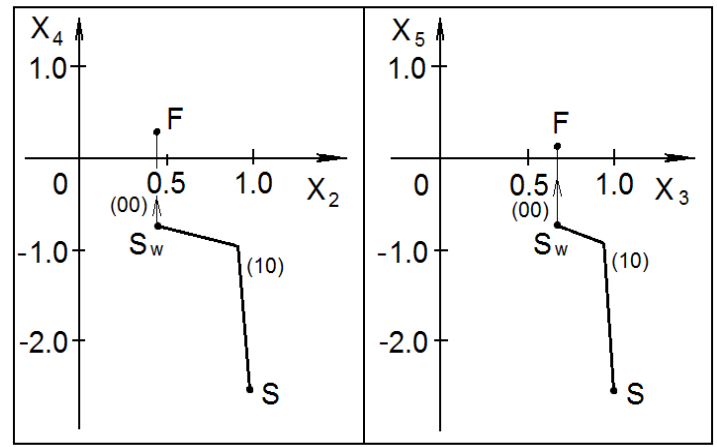

Fig. 7 Projections of trajectory of optimization process for other correct value of $\boldsymbol{\Psi}_{0 c}=(0.1,-0.02,-0.5,-0.2,0.6)$

It is important to note that the strategy found from the conditions of the maximum principle and corresponding to the control vector with two parts (10) and (00) and switching between them on a 73rd step of integration has not been predicted in previous research. In earlier executed analysis it was supposed that the optimum strategy must be constructed on the basis of the combination of MTSO and TSO. This assumption is not always fulfiled as shown in the present analysis.

This example shows that earlier predicted optimum structure consisting of only of MTSO and TSO is not always optimum.

Let's consider use of the maximum principle for the problem of optimization of an active circuit the three-cascade transistor amplifier that is displayed in Fig8.

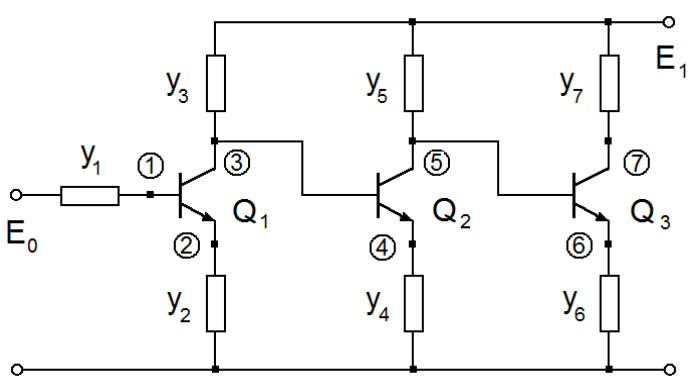

Fig. 8 Three-cascade amplifier 
The sources of voltage of $E_{0}, E_{1}$ are defined. The model of the transistor is the known model of EbersMoll on a direct current used in the SPICE system, is chosen [28]. The three-cascade amplifier is characterized by seven independent variables $y_{1}, y_{2}, y_{3}, y_{4}, y_{5}, y_{6}, y_{7}(K=7)$ and seven dependent variables $V_{1}, V_{2}, V_{3}, V_{4}, V_{5}, V_{6}, V_{7}(M=7)$. The vector $X$ includes 14 components and can be defined by the following formulas: $x_{1}^{2}=y_{1}, \quad x_{2}^{2}=y_{2}, \quad x_{3}^{2}=y_{3}$, $x_{4}^{2}=y_{4}, \quad x_{5}^{2}=y_{5}, \quad x_{6}^{2}=y_{6}, \quad x_{7}^{2}=y_{7}, \quad x_{8}=V_{1}$, $x_{9}=V_{2}, x_{10}=V_{3}, x_{11}=V_{4}, x_{12}=V_{5}, x_{13}=V_{6}$, $x_{14}=V_{7}$.

The objective function was determined as a sum of squares of differences between the specified and current values of voltages across the transistor transitions for all transistors by means of following formula:

$$
C(\mathbf{X})=\sum_{i=1}^{3}\left[\left(V_{E B i}-V_{E B 0 i}\right)^{2}+\left(V_{C B i}-V_{C B O i}\right)^{2}\right],
$$

where $V_{E B i}$ and $V_{C B i}$ are the current voltages on the emitter and collector junctions for all the transistors, respectively, and $V_{E B O i}$ and $V_{C B O i}$ are the given voltages on the emitter and collector junctions, respectively. These parameters are set as follows: $V_{E B O 1}=-0.3 \mathrm{~V}, V_{C B O 1}=5.4 \mathrm{~V}, V_{E B O 2}=-0.3 \mathrm{~V}, V_{C B O 2}=6.5$ $\mathrm{V}, V_{E B O 3}=-0.35 \mathrm{~V}$ and $V_{C B O 3}=6.6 \mathrm{~V}$. This gives us an amplification of 45,000 or higher.

The control vector $\mathbf{U}$ consists of seven control functions: $\quad \mathbf{U}=\left(u_{1}, u_{2}, u_{3}, u_{4}, u_{5}, u_{6}, u_{7}\right) . \quad$ The mathematical model of a circuit is defined by the system of seven equations $(M=7)$ and the optimization procedure (5) includes 14 equations. The total structural basis contains $2^{M}=128$ different optimization strategies.

The final values of the independent variables that minimise the cost function (20) are next: $x_{1}=0.01251, x_{2}=0.0812, x_{3}=0.0615, x_{4}=0.094$, $x_{5}=0.086, x_{6}=0.234, x_{7}=0.206$. These values were obtained for all analysed strategies.

The results for TSO and some other strategies having the CPU time less than for TSO are presented below in Table 2 .

The fastest strategy is MTSO with the control vector of (1111111) which has a processor time of 3.91 seconds and corresponds to the acceleration of the optimization process in 707 times in comparison with TSO. However, applying the maximum principle we obtained bigger acceleration.
Table 2. Data of some strategies of structural basis.

\begin{tabular}{|c|c|c|c|}
\hline $\mathrm{N}$ & Control & Iterations & Total CPU \\
\hline & vector & number & time $(\mathrm{sec})$ \\
\hline & $\left(\begin{array}{llllllll}0 & 0 & 0 & 0 & 0 & 0 & 0\end{array}\right)$ & 1907780 & 2766.28 \\
\hline & $\left(\begin{array}{lllllll}0 & 0 & 1 & 1 & 1 & 0 & 0\end{array}\right)$ & 131671 & 145.72 \\
\hline & $\left(\begin{array}{llllllll}0 & 0 & 1 & 1 & 1 & 0 & 1\end{array}\right)$ & 90328 & 75.26 \\
\hline & $4(0110111)$ & 28213 & 17.21 \\
\hline & $5(1011111)$ & 37949 & 11.88 \\
\hline & $(11110111)$ & 62534 & 19.55 \\
\hline & $7(1111110)$ & 103802 & 47.95 \\
\hline & $3(1111111)$ & 47079 & 3.91 \\
\hline
\end{tabular}

Applying the methodology of the maximum principle based on formulas (10)-(18), we analyzed the behaviour of the function of Hamilton for the strategies in Table 2.

The condition (19) is transformed for this example to the following expression:

$$
\begin{aligned}
& H_{\max }(\mathbf{X}, \boldsymbol{\Psi})=h_{c}(\mathbf{X}, \boldsymbol{\Psi})+ \\
& +\max \left\{\begin{array}{l}
h_{v}(\mathbf{X}, \boldsymbol{\Psi},(0,0,0,0,0,0,0)), h_{v}(\mathbf{X}, \boldsymbol{\Psi},(0,0,0,0,0,0,1)), \\
\ldots, h_{v}(\mathbf{X}, \boldsymbol{\Psi},(1,1,1,1,1,1,0)), h_{v}(\mathbf{X}, \boldsymbol{\Psi},(1,1,1,1,1,1,1))
\end{array}\right\}
\end{aligned}
$$

The vectors of $\mathbf{X}$ and $\boldsymbol{\Psi}$ consist of 14 components for this example. The behaviour of the function of Hamilton on an interval from 0 to 5.5 seconds for six different values of the control vector from Table 2 is presented in Fig9.

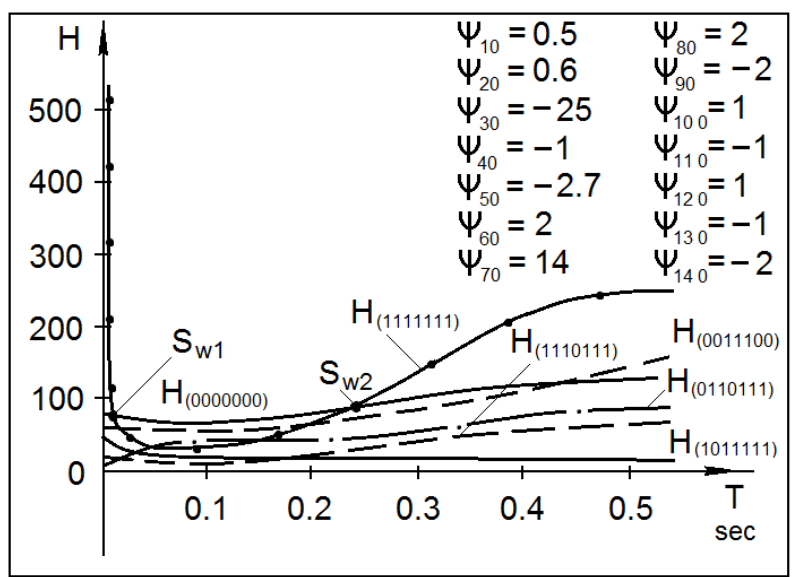

Fig. 9 Time dependency of functions $H_{(0000000)}$, $H_{(0011100)}, H_{(011011)}, H_{(1011111)}, H_{(111011)}, H_{(111111)}$ for other correct value of parameter $\boldsymbol{\Psi}_{0 c}$

The correct initial value of an auxiliary vector $\boldsymbol{\Psi}, \quad$ is defined as: $\Psi_{0 c}=(0.5,0.6,-25,-1,-2.7,2,14,2,-2,1,-1,1,-1,-2)$.

This value has been obtained by the additional optimizing procedure for the following initial point 
of the designing process of $\mathbf{X}^{0}:\left(x_{1,0}=0.1, x_{2,0}=1\right.$, $x_{3,0}=0.316, x_{4,0}=0.316, x_{5,0}=1, x_{6,0}=0.105$, $x_{7,0}=0.5, x_{8,0}=-2, x_{9,0}=1, x_{10,0}=-20, x_{11,0}=2$, $\left.x_{12,0}=-20, x_{13,0}=2, x_{14,0}=-20\right)$.

The Hamilton function $H_{(111111)}$ for strategy (1111111) has the greatest value of all analyzed strategies on the initial part of optimization process from 0 to a point of $S_{\mathrm{w} 1}$, which corresponds to the 29th step of integration of the system (5).

In this point, the Hamiltonian $H_{(0000000)}$, which corresponds to TSO, is equal to $H_{(111111)}$ and then is greater than for all other strategies. The Hamiltonian $H_{(0000000)}$ remains maximum on the interval since a point $S_{\mathrm{w} 1}$ to a point $S_{\mathrm{w} 2}$., which corresponds to the 200th step. Then, since the point $S_{\mathrm{w} 2}$ the Hamiltonian $H_{(1111111)}$ is greater than for all other strategies once again and remains so until the end of the optimization process. The full time of the optimization process is equal to 0.55 seconds. Acceleration for the obtained optimum strategy is equal to 5,029 times in comparison with TSO.

The points of switching $S_{\mathrm{w} 1}$ and $S_{\mathrm{w} 2}$ appear automatically in the numerical algorithm by means of comparing the functions of Hamilton for all strategies. So, we can state that the maximum principle can be base for searching of the optimization process with the minimal CPU time.

Summarising the obtained results, it is possible to indicate two important facts. First, the theoretical result is obtained - theoretical justification is given for the earlier discovered effect of acceleration of the process of circuit optimization in the conditions of a new methodology of design. This justification is based on the maximum principle.

Second, the analysis of the optimization process of the presented circuits showed that application of the maximum principle really allows for the finding of the optimum structure of the control vector $\mathbf{U}(\mathrm{t})$ by means of an iterative procedure. Thus, considerable reduction of CPU time in comparison with traditional approach is observed when using the maximum principle. This result is obtained for the case of $\mathrm{N}$-dimensional space of parameters.

\section{Conclusion}

Analysis of the application of maximum principle to a problem of circuit optimization proves that the formerly studied effect of acceleration on the process of optimization appears owing to this principle. This means that the maximum principle of Pontryagin provides a theoretical justification for the acceleration effect that appears when we use the generalized formulation of process of circuit optimization. It is confirmed that the maximum principle allows for finding one or several local minima of the functional that is defined as the processor time. Aside from that, the use of the maximum principle provides the chance to significantly reduce the computing time for circuit optimization.

The analysis of optimization process of the presented circuits showed that application of the maximum principle really allows finding the optimum structure of the control vector $\mathbf{U}(\mathrm{t})$ by means of iterative procedure. These results were obtained for $\mathrm{N}$-dimensional space of parameters.

\section{References:}

[1] O. Osterby, and Z. Zlatev, Direct methods for sparse matrices, Springer-Verlag, N.Y., 1983.

[2] A. George, On Block Elimination for Sparse Linear Systems, SIAM J. Numer. Anal., Vol.11, No.3, 1984, pp. 585-603.

[3] N. Rabat, A.E. Ruehli, G.W. Mahoney, and J.J. Coleman, A survey of macromodelling. IEEE Int. Symp. on Circuits and Systems, 1985, pp. 139-143.

[4] M. Tadeusiewicz, and A. Kuczynski, A very fast method for the DC analysis of diodetransistor circuits, Circuits Systems and Signal Processing, Vol.32, No.3, 2013, pp. 433-451.

[5] R.K. Brayton, G.D. Hachtel, and A.L. Sangiovanni-Vincentelli, A survey of optimization techniques for integrated-circuit design, Proceedings IEEE, Vol.69, No.10, 1981, pp. 1334-1362.

[6] A.E. Ruehli (Ed.), Circuit analysis, simulation and design Part 2, Elsevier Science Publishers, Amsterdam, 1987.

[7] G. Stehr, M. Pronath, F. Schenkel, H. Graeb, and K. Antreich, Initial sizing of analog integrated circuits by centering within topology-given implicit specifications, Proc. of the IEEE/ACM Int. Conf. CAD, 2003, pp. 241246.

[8] M. Hershenson, S. Boyd, and T. Lee, Optimal design of a CMOS op-amp via geometric programming, IEEE Trans. CAD of Integrated Circuits and Systems, Vol.20, No.1, 2001, pp. $1-21$.

[9] D. Nam, Y. Seo, L. Park, C. Park, and B. Kim, Parameter optimization of an on-chip voltage reference circuit using evolutionary programming. IEEE Trans. Evolutionary Comp., Vol.5, 2001, pp. 414-421.

[10] N.F. Paulino, J. Goes, and A. Steiger-Garcao, Design methodology for optimization of analog building blocks using genetic 
algorithms. Proc. Symposium Circuits and Systems, 2001, pp. 435-438.

[11] Alpaydin, G., Balkir, S., \& Dundar, G. (2003). An evolutionary approach to automatic synthesis of high performance analog integrated circuits. IEEE Transactions on Evolutionary Comp., 7, 240-252.

[12] A. Srivastava, T. Kachru, and D. Sylvester, Low-Power-Design Space Exploration Considering Process Variation Using Robust Optimization, IEEE Trans. CAD of Integrated Circuits, Vol.26, No.1, 2007, pp. 67-79.

[13] B. Liu, Y. Wang, Z. Yu, L. Liu, M. Li, Z. Wang, J. Lu, and F.V. Fernandez, Analog circuit optimization system based on hybrid evolutionary algorithms. Integration the VLSI J., Vol.42, No.2, 2009, pp. 137-148.

[14] F. Yengui, L. Labrak, F. Frantz, R. Daviot, N. Abouchi, and I. O'Connor, A hybrid GA-SQP algorithm for analog circuits sizing, circuits and systems, Circuits and Systems, Vol.3, 2012, pp. 146-152.

[15] M.R.M. Ridzuan, E.E. Hassan, A.R. Abdullah, N. Bahaman, and A.F.A. Kadir, A new meta heuristic evolutionary programming (NMEP) in optimizing economic energy dispatch, $J$. Telecomm. Electron. Comp. Engineer., Vol.8, No.2, 2016, pp. 35-40.

[16] J. Robinson, and Y. Rahmat-Samii, Particle swarm optimization in electromagnetic. IEEE Trans. Antennas and Propagation, Vol.52, No.2, 2004, pp. 397-407.

[17] M.A. Zaman, M. Gaffar, M.M. Alam, S.A. Mamun, and M.A. Matin, Synthesis of antenna arrays using artificial bee colony optimization algorithm, Int. J. of Micro. and Optic. Technol., Vol.6, No.8, 2011, pp. 234-241.

[18] I.S. Kashirskiy, Y.K. Trokhimenko, General optimization for electronic circuits, Tekhnika, Kiev, 1979.

[19] V. Rizzoli, A. Costanzo, and C. Cecchetti, Numerical optimization of broadband nonlinear microwave circuits, Proceedings of IEEE MTT-S Int. Symposium, Vol.1, 1990, pp. 335-338.

[20] E.S. Ochotta, R.A. Rutenbar, and L.R. Carley, Synthesis of high-performance analog circuits in ASTRX/OBLX, IEEE Trans. CAD Integrated Circuits and Systems, Vol.15, No.3, 1996, pp. 273-294.

[21] A. Zemliak, Analog circuit optimization on basis of control theory approach, COMPEL: The Int. J. Computation and Mathematics in Electrical and Electronic Engineering, Vol.33, No.6, 2014, pp. 2180-2204.
[22] L.S. Pontryagin, V.G. Boltyanskii, R.V. Gamkrelidze, and E.F. Mishchenko, The mathematical theory of optimal processes, Interscience Publishers, Inc., N.Y., 1962.

[23] L.W. Neustadt, Synthesis of time-optimal control systems. J. Math. Analysis and Appl., Vol.1, 1960, pp. 484-492.

[24] J.B. Rosen, Iterative solution of nonlinear optimal control problems, J. SIAM, Control Series A, 1966, pp. 223-244.

[25] R.P. Fedorenko, Approximate Solution of Optimal Control Problems, Nauka, Moscow, 1978.

[26] L. Bourdin, and E. Trélat, Pontryagin maximum principle for finite dimensional nonlinear optimal control problems on time scales, SIAM J. Control Optim., Vol.51, No.5, 2013, pp. 3781-3813.

[27] A. Zemliak, Maximum principle for problem of circuit optimization. Electronics Letters, Vol.52, No.9, 2016, pp. 695-697.

[28] G. Massobrio, and P. Antognetti, Semiconductor Device Modeling with SPICE, McGraw-Hill Inc., N.Y., 1993.

\section{Creative Commons Attribution License 4.0 (Attribution 4.0 International, CC BY 4.0)}

This article is published under the terms of the Creative Commons Attribution License 4.0

https://creativecommons.org/licenses/by/4.0/deed.en US 